\title{
Mesenchymal stromal cell application as an emerging translational medicine for acute respiratory distress syndrome
}

\author{
Hyun Jung Lee ${ }^{1}$, Won-Young Kim $^{2}$ \\ ${ }^{1}$ Department of Anatomy and Cell Biology, ${ }^{2}$ Department of Internal Medicine, Chung-Ang University Hospital, Chung-Ang University College of \\ Medicine, Seoul, Korea \\ Correspondence to: Won-Young Kim, MD, PhD. Department of Internal Medicine, Chung-Ang University Hospital, Chung-Ang University College \\ of Medicine, 102 Heukseok-ro, Dongjak-gu, Seoul 06973, Korea. Email: wykim81@cau.ac.kr; steve8126@hanmail.net. \\ Provenance and Peer Review: This article was commissioned by the Editorial Office, Annals of Translational Medicine. The article did not undergo \\ external peer review. \\ Comment on: Jung YJ, Park YY, Huh JW, et al. The effect of human adipose-derived stem cells on lipopolysaccharide-induced acute respiratory \\ distress syndrome in mice. Ann Transl Med 2019;7:674.
}

Submitted Feb 07, 2020. Accepted for publication Feb 28, 2020.

doi: $10.21037 /$ atm.2020.02.82

View this article at: http://dx.doi.org/10.21037/atm.2020.02.82

Acute respiratory distress syndrome (ARDS) is a lifethreatening disease in critically ill patients, in whom it is associated with a mortality rate of $30-45 \%$ (1). The prevalence of ARDS in the intensive care unit (ICU) is high and is responsible for high morbidity and mortality rates among mechanically ventilated patients, although current therapies for ARDS are only symptomatic. Management strategies include protective mechanical ventilation and fluid-restrictive strategies, which aim to minimize symptoms while providing organ support. Therefore, clinicians and scientists hope for new pharmacologic, biologic, and genetic strategies to further our understanding of the pathogenesis, pathophysiology, and treatment of ARDS, which will improve clinical outcomes.

Cell therapy is one potential strategy that has shown great promise in preclinical ARDS studies. Multiple studies have revealed immunomodulatory and anti-inflammatory effects after treatment using mesenchymal stromal cells (MSCs). These non-hematopoietic multipotent stromal precursor cells can be isolated from various tissues, including the bone marrow, adipose tissue, dental pulp, placental tissue, cord blood, and matrix (2). Given the ease of isolating these cells, it was originally thought that delivery of cultured MSCs to the injured tissues would result in migration and differentiation into a locally appropriate phenotype and function, thereby leading to tissue repair (3). However, MSCs exhibit low engraftment into the injured tissues in some cases, and several animal studies clearly showed that injected MSCs could repair damaged tissue and lead to functional recovery without differentiating into the specialized cells for that tissue (4). Thus, it appears that MSCs do not typically differentiate into tissue-specific specialized cells but rather exert their regenerative ability through a paracrine effect. For example, MSCs may contribute to the resolution of inflammation through immunomodulatory and anti-inflammatory mechanisms, such as the release of soluble factors, including nitric oxide, indoleamine 2,3-dioxygenase, prostaglandin E2 (PGE2), and interleukin-10 (IL-10) $(5,6)$. Thus, despite a lack of clarity regarding their specific molecular mechanisms, MSCs remain an attractive therapeutic candidate for treating acute or chronic inflammation-related diseases. Moreover, MSCs have low immunogenicity that allows for safe use in allogenic donor-matched settings or even xenogeneic settings, which suggests that they have many clinical advantages.

Jung et al. recently published a report in the Annals of Translational Medicine describing their mouse model of ARDS that could be induced using intratracheal administration of lipopolysaccharide (LPS) (7). These mice were then treated with human adipose-derived stem cells (ASCs) that were injected intravenously at $4 \mathrm{~h}$ after ARDS induction. Eggenhofer et al. have reported that intravenously infused MSCs are short-lived and mostly trapped in the lungs at $1 \mathrm{~h}$ after the infusion, with few MSCs observed in the other organs (6). Moreover, at $24 \mathrm{~h}$ 
after the MSC infusion, most MSCs were dead and were detected in the liver, which suggests that an infusion of MSCs might be useful for targeting acute lung injuries, such as ARDS, and they might effectively reduce inflammation of the injured lung.

Jung et al. also showed that injected ASCs attenuated the alveolar hemorrhage and congestion that was induced by the intrapulmonary administration of LPS, which lowered the overall lung injury scores relative to the LPS control group (7). Many studies examining the effect of MSCs in lung injury models have also indicated that MSC application was associated with reduced lung injury and a recovery of lung function. For example, rats with ventilator-induced lung injury (VILI) received a tracheal administration of rat bone marrow-derived mesenchymal stromal cells (BMSCs) and subsequently experienced recovery of their lung function (8). Intravenous injection of human BMSCs into rats with acute lung injury also accelerated their lung recovery and bacterial clearance (9), which indicates that MSCs provide regenerative effects independent of the species from which they were derived. However, no changes in infiltrating neutrophils or inflammatory cytokine levels (e.g., IL-1 $\beta$, IL-6, and TNF- $\alpha$ ) were observed after the injection of ASCs in the study by Jung et al., although the lung injury scores did recover. Nevertheless, these assays were performed on days 2 and 7 after ARDS induction, while inflammatory cytokine level (e.g., TNF- $\alpha$ and IL-6) tend to rapidly respond to acute systemic inflammation or the induction of experimental endotoxemia. In the plasma of 10 young healthy volunteers, notable TNF- $\alpha, \operatorname{IFN}-\gamma$, and IL-6 levels appeared within the first hour after an intravenous bolus injection of LPS (10). In this context, TNF- $\alpha$ tends to exhibit a monophasic peak after approximately $90 \mathrm{~min}$, whereas IL-6 and IFN- $\gamma$ levels tend to peak after approximately $120 \mathrm{~min}$ and then gradually decrease (11). Thus, detecting cytokine levels earlier than day 2 or especially day 7 might have revealed significant differences between the LPS and LPS/ASCs groups in the study by Jung et al. Gonzalez-Rey et al. demonstrated that human ASCs could significantly reduce the levels of TNF- $\alpha$, IL- $1 \beta$, IFN- $\gamma$, and IL- 6 starting at $18 \mathrm{~h}$ after cecal ligation and puncture (CLP) in a mouse model (12). Pedrazza $e t a l$. also used a CLP mouse model to determine that mouse ASCs could decrease the levels of TNF- $\alpha$ and IL-6 at $12 \mathrm{~h}$ after sepsis induction (13).

The findings described above might indicate that the therapeutic effect of MSCs is dependent on the dose (i.e., high or low doses in one or several treatments) and the timing of their administration (early or later after disease onset). The study by Jung et al. revealed that a single dose of $2 \times 10^{5}$ human ASCs at $4 \mathrm{~h}$ after sepsis induction improved the lung injury score, although there was no information regarding the survival rate after ASC administration. Gonzalez-Rey et al. reported that mice administered a single intraperitoneal dose of $1 \times 10^{6}$ human ASCs at $30 \mathrm{~min}$ after LPS injection had a better survival rate than those administered a single dose of $3 \times 10^{5}$ cells (12). Hall et al. used three intravenous administrations of mouse BMSCs after CLP induction $\left(5 \times 10^{5}\right.$ at $2 \mathrm{~h}, 2.5 \times 10^{5}$ at $24 \mathrm{~h}$, and $2.5 \times 10^{5}$ at $48 \mathrm{~h}$ ), which also improved the mortality rate among mice (14). While there are no direct comparisons of single-dose and repeated-dose treatments, these results suggest that multiple doses or a single early dose might improve the outcomes among mice and enhance their likelihood of recovering from sepsis. Nevertheless, further studies are needed to clarify the optimal dose(s) and timing of administration(s) in this setting.

Another important issue is the cell source, as MSCs can be harvested from various adult and neonatal tissues. Clinical studies have widely used ASCs and umbilical cord bloodderived MSCs (UC-MSCs) because they are readily available, although animal studies have tended to use BMSCs, which were the first described type of cells and considered the gold standard for MSCs (15). It is possible that the cell source might affect MSC efficacy, although standard methods have been described for the isolation, culture, and characterization of MSCs. Bochev et al. have also compared the antiinflammatory effects of human ASCs and human BMSCs, based on immunoglobulin (Ig) produced by peripheral blood mononuclear cells (PBMCs) after stimulation using pokeweed mitogen (16). The Ig production was significantly reduced by MSC treatment, with ASCs providing greater suppression than BMSCs. However, Elman et al. reported that BMSCs appeared to provide greater anti-inflammatory effects and improved mouse survival better than ASCs in a model of LPS-induced systemic inflammation (17). Thus, it is difficult to conclude which MSC source is the most appropriate, although human ASCs appear to be more genetically and morphologically stable in long-term cultures, with a lower senescence ratio, a higher proliferative capacity, and a greater retention of differentiation potential (vs. human BMSCs) (18). Furthermore, approximately 500-fold more ASCs can be obtained from adipose tissue than the amount of BMSCs isolated from an equivalent amount of bone marrow stroma (19). Therefore, ASCs may be easier to obtain and a more 
effective option for anti-inflammatory therapy. Moreover, Bernardo et al. suggested that ASCs could be clinically used without expansion after harvest (20), which would presumably be safer and more effective than cultured cells used after ex vivo manipulations that potentially lead to the accumulation of genetic and epigenetic alterations. It is possible that Jung et al. might have observed substantially better improvements in lung function and inflammatory cytokine levels if they had used freshly prepared human ASCs in their model.

In conclusion, the results reported by Jung et al. suggest that human ASCs may be useful for treating ARDS in a mouse model (based on improvement in the lung injury score), which might make them a readily accessible option for clinical applications. However, that study was limited by the fact that the ASCs were used after several days of culture expansion, and the biochemical assays were performed relatively late after ARDS induction. These issues may have reduced the efficacy of ASCs as a therapeutic strategy for ARDS, and further studies are needed to clarify the potential clinical utility of human ASCs for treating ARDS.

\section{Acknowledgments}

Funding: None.

\section{Footnote}

Conflicts of Interest: The authors have no conflicts of interest to declare.

Ethical Statement: The authors are accountable for all aspects of the work in ensuring that questions related to the accuracy or integrity of any part of the work are appropriately investigated and resolved.

Open Access Statement: This is an Open Access article distributed in accordance with the Creative Commons Attribution-NonCommercial-NoDerivs 4.0 International License (CC BY-NC-ND 4.0), which permits the noncommercial replication and distribution of the article with the strict proviso that no changes or edits are made and the original work is properly cited (including links to both the formal publication through the relevant DOI and the license). See: https://creativecommons.org/licenses/by-nc-nd/4.0/.

\section{References}

1. Máca J, Jor O, Holub M, et al. Past and Present ARDS Mortality Rates: A Systematic Review. Respir Care 2017;62:113-22.

2. Chamberlain G, Fox J, Ashton B, et al. Concise review: mesenchymal stem cells: their phenotype, differentiation capacity, immunological features, and potential for homing. Stem Cells 2007;25:2739-49.

3. Barry FP, Murphy JM. Mesenchymal stem cells: clinical applications and biological characterization. Int $\mathrm{J}$ Biochem Cell Biol 2004;36:568-84.

4. Di Matteo B, El Araby MM, D'Angelo A, et al. AdiposeDerived Stem Cell Treatments and Formulations. Clin Sports Med 2019;38:61-78.

5. Diaz MF, Vaidya AB, Evans SM, et al. Biomechanical Forces Promote Immune Regulatory Function of Bome Marrow Mesenchymal Stromal Cells. Stem Cells 2017;35:1259-72.

6. Eggenhofer E, Benseler V, Kroemer A, et al. Mesenchymal stem cells are short-lived and do not migrate beyond the lungs after intravenous infusions. Front Immunol 2012;3:297.

7. Jung YJ, Park YY, Huh JW, et al. The effect of human adipose-derived stem cells on lipopolysaccharide-induced acute respiratory distress syndrome in mice. Ann Transl Med 2019;7:674.

8. Curley GF, Ansari B, Hayes M, et al. Effects of intratracheal mesenchymal stromal cell therapy during recovery and resolution after ventilator-induced lung injury. Anesthesiology 2013;118:924-32.

9. Devaney J, Horie S, Masterson C, et al. Human mesenchymal stromal cells decrease the severity of acute lung injury induced by E.coli in the rat. Thorax 2015;70:625-35.

10. Taudorf S, Krabbe KS, Berg RM, et al. Human models of low-grade inflammation: bolus versus continuous infusion of endotoxin. Clin Vaccine Immunol 2007;14:250-5.

11. Andreasen AS, Krabbe KS, Krogh-Madsen R, et al. Human Endotoxemia as a Model of Systemic Inflammation. Curr Med Chem 2008;15:1697-705.

12. Gonzalez-Rey E, Anderson P, González MA, et al. Human adult stem cells derived from adipose tissue protect against experimental colitis and sepsis. Gut 2009;58:929-39.

13. Pedrazza L, Lunardelli A, Luft C, et al. Mesenchymal 
stem cells decrease splenocytes apoptosis in a sepsis experimental model. Inflamm Res 2014;63:719-28.

14. Hall SR, Tsoyi K, Ith B, et al. Mesenchymal stromal cells improve survival during sepsis in the absence of heme oxygenase-1: the importance of neutrophils. Stem Cells 2013;31:397-407.

15. Friedenstein AJ, Gorskaja JF, Kulagina NN. Fibroblast precursors in normal and irradiated mouse hematopoietic organs. Exp Hematol 1976;4:267-74.

16. Bochev I, Elmandjian G, Kyurkchiev D, et al. Mesenchymal stem cells from human bone marrow or adipose tissue differently modulate mitogen-stimulated B-cell immunoglobulin production in vitro. Cell Biol Int 2008;32:384-93.

Cite this article as: Lee HJ, Kim WY. Mesenchymal stromal cell application as an emerging translational medicine for acute respiratory distress syndrome. Ann Transl Med 2020;8(6):267. doi: 10.21037/atm.2020.02.82
17. Elman JS, Li M, Wang F, et al. A comparison of adipose and bone marrow-derived mesenchymal stromal cell secreted factors in the treatment of systemic inflammation. J Inflamm (Lond) 2014;11:1.

18. Strioga M, Viswanathan S, Darinskas A, et al. Same or Not the Same? Comparison of Adipose Tissue-Derived Versus Bone Marrow-Derived Mesenchymal Stem and Stromal Cells. Stem Cells Dev 2012;21:2724-52.

19. Hass R, Kasper C, Böhm S, et al. Different populations and sources of human mesenchymal stem cells (MSC): A comparison of adult and neonatal tissue-derived MSC. Cell Commun Signal 2011;9:12.

20. Bernardo ME, Locatelli F, Fibbe WE. Mesenchymal stromal cells. Ann N Y Acad Sci 2009;1176:101-17. 